Questions vives

\section{Questions Vives}

Recherches en éducation

Vol. $9 n^{\circ} 19$ | 2013

Éducation routière, changement de comportement et formation à la conduite : constat, enjeux et transformations

\title{
Éducation routière, changement de comportement et formation à la conduite : constats, enjeux et transformations
}

Driver education, change of behaviour and driver training. Findings, issues and transformations

\section{Christine Poplimont}

\section{(2) OpenEdition}

Journals

Édition électronique

URL : http://journals.openedition.org/questionsvives/1315

DOI : $10.4000 /$ questionsvives. 1315

ISBN : 978-2-8218-1394-6

ISSN : $1775-433 \mathrm{X}$

Éditeur

Université Aix-Marseille (AMU)

Édition imprimée

Date de publication : 15 juillet 2013

Pagination : 15-19

ISBN : 978-2-912643-43-8

ISSN : 1635-4079

\section{Référence électronique}

Christine Poplimont, «Éducation routière, changement de comportement et formation à la conduite: constats, enjeux et transformations », Questions Vives [En ligne], Vol.9 n¹9 | 2013, mis en ligne le 15 octobre 2013, consulté le 22 septembre 2020. URL : http://journals.openedition.org/questionsvives/ 1315 ; DOI : https://doi.org/10.4000/questionsvives.1315

Ce document a été généré automatiquement le 22 septembre 2020.

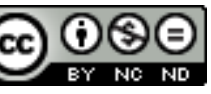

Questions Vives est mis à disposition selon les termes de la licence Creative Commons Attribution Pas d'Utilisation Commerciale - Pas de Modification 4.0 International. 


\title{
Éducation routière, changement de comportement et formation à la conduite : constats, enjeux et transformations
}

\author{
Driver education, change of behaviour and driver training. Findings, issues and \\ transformations
}

Christine Poplimont

1 La lutte contre l'insécurité routière est l'une des priorités du gouvernement français et des pouvoirs publics depuis 2002. En effet, le risque routier constitue aujourd'hui le premier risque d'accident mortel au travail et reste la première cause de mortalité pour les moins de vingt-cinq ans. Cette lutte s'appuie sur le renforcement des sanctions et sur le développement d'une politique d'éducation et de prévention du risque routier au bénéfice de l'ensemble des usagers de la route. Mais, malgré les progrès exceptionnels observés depuis 2003, on observe un ralentissement de la diminution du nombre de morts. Cette inflexion dans la courbe de mortalité nous incite à envisager des solutions complémentaires. En effets la lutte contre l'insécurité routière, s'est axée en France, sur les principes behavioristes de renforcements négatifs (sanctions pécuniaires ; perte de points, voire du permis de conduire...), et très peu sur le renforcement positif de comportements routiers adaptés et apaisés. Par ailleurs, il y a très peu de moyens mis en place pour permettre aux usagers de la route de continuer à se former tout au long de leur vie de conducteur.

2 L'éducation et la formation pourraient influencer la régulation des comportements à risque, et ainsi permettre de développer des compétences (Le Boterf, 2000) autoévaluatives (Vial, 1997) permettant à chaque usager de passer d'un état agentique (Joule-Beauvois, 1990) à un état d'acteur, voire d'auteur (Ardoino \& Berger, 1986) de leurs déplacements. L'éducation faisant partie intégrante de la construction identitaire de l'être humain, elle soulève notamment les notions de savoirs, savoir-faire, de savoirêtre, mais aussi de savoir-devenir. L'importance de l'éducabilité réside, donc aussi, dans 
un souci de cohérence nécessaire au bon fonctionnement des hommes entre eux. Mais plus important encore, nos apprentissages ne sont pas figés. Pour cela, la volonté de progression de chacun résulte de sa potentialité à changer. Ainsi, considérons-nous l'apprentissage de la conduite automobile comme relevant en partie d'un processus de socialisation. Cette socialisation peut être définie «comme l'appropriation et l'intériorisation actives par l'individu des valeurs, des attitudes, des représentations des règles sociales et de normes de références sociales... » (Vurpillot \& Pêcheux, 1992) permettant une autonomie et une adaptation à la circulation routière.

3 Mais la socialisation par l'Éducation routière suppose à la fois des compétences cognitives, perceptives, sociales, affectives et auto-évaluatives... À l'aube de grands bouleversements (réformes des métiers de l'enseignement de la conduite automobile et de la Sécurité Routière, intégration de la matrice GDE (GADGET, 1999) dans les nouveaux référentiels et programmes de formation, renforcement du continuum éducatif...), l'Éducation Routière se doit de réfléchir à une politique globale et efficiente de l'enseignement de la Sécurité Routière.

4 À travers cette revue de question, divers auteurs, chercheurs et enseignants nous proposent des pistes d'améliorations et de réflexions de nos pratiques.

5 1. Comme nous l'avons vu précédemment, les jeunes de 18 à 24 ans paient un lourd tribut à l'accidentologie routière. En 2011, alors qu'ils ne représentaient que $9 \%$ de la population française, ils représentaient $21 \%$ des tués (ONISR, 2011). Pour J.S. Wilde, la réduction de l'accidentalité des jeunes conducteurs passe par un travail sur la perception du risque et par la réduction de son acceptation. En effet les conducteurs novices acceptent un niveau de risque plus élevé que les autres conducteurs et se mettent plus souvent dans des situations dangereuses. J.S. Wilde, plus connu pour l'élaboration de la théorie homéostasique du risque routier (Wilde, 1982, 1988, 2001), nous montre ici qu'une approche centrée sur la récompense et le renforcement positif est à développer dans l'apprentissage de la conduite automobile. En effet, le résultat des expériences des programmes incitatifs, montrent que la valorisation des comportements sécuritaires (par l'argent notamment), permet une réduction significatrice de l'accidentologie. Ainsi son travail nous permet-il de nous éclairer sur des pratiques innovantes à mettre en œuvre dans l'Éducation Routière.

6 2. Jean-Pascal Assailly, chercheur à l'IFSTTAR, spécialiste de l'Éducation Routière, nous livre son analyse au travers du champ théorique et méthodologique proposé par la matrice GDE. Jean-Pascal Assailly passe en revue les différentes formations avant et post permis. Il met l'accent sur les pédagogies innovantes pour traduire le sur risque des jeunes conducteurs novices. Les recherches montrent les différentes stratégies mises en œuvre, en y associant leurs évaluations en termes d'efficience. Le permis graduel, stratégie dominante dans les pays industrialisés, y est défini comme le système d'accès à la conduite, le plus efficace. On constate dans les pays l'ayant mis en place, des diminutions significatives des taux d'accidents.

7 Une autre voie nous est présentée à travers les différents niveaux de la matrice GDE. Cette matrice structuraliste nous propose une autre approche en privilégiant celles qui sont centrées sur les attitudes et la personnalité de chacun, plutôt que de travailler sur les apprentissages d'automatismes à la conduite automobile. L'autoévaluation y est présentée aussi comme apportant des résultats prometteurs (Gregersen, 1996).

8 3. Après avoir fait l'historique de l'Éducation routière, Marc Camiolo, docteur en sociologie, en atteste son échec en tant que moyen de réguler le phénomène de 
l'insécurité routière. Marc Camiolo y décrit le risque et son acceptabilité comme une production collective et culturelle de notre société. De plus, à partir d'analyses d'accidents de la circulation dans le cadre de leçons de conduite, Marc Camiolo démontre ainsi que les stéréotypes et les valeurs transmises par la société influent sur l'enseignement de la conduite automobile et de la sécurité routière. Il y démontre, par ailleurs, que notre société patriarcale transmet des valeurs de courage et de virilité qui sont présentes dans nos apprentissages. Ainsi pour Marc Camiolo, le système étatique de l'apprentissage de la conduite transmet des normes sociales et culturelles valorisant une certaine forme de prise de risque. Il y voit là une cause, expliquant le peu d'efficience des formations ayant comme objet la réduction des risques routiers.

4. Quelques soient les activités risquées ou dangereuses, on observe, dans nos sociétés industrialisées, une surimplication du sexe masculin. À partir des dernières recherches en cours sur le sujet, Marie-Axelle Granié, chercheure à l'IFSTTAR, démontre, dans son article, que la différence d'accidentalité entre les hommes et les femmes est davantage due à un principe de conformité aux stéréotypes de sexe et de conformité aux règles qu'à une exposition aux situations à risque. Elle voit la conduite automobile comme une activité stéréotypée où le genre et les stéréotypes sont le reflet de notre socialisation. À partir d'une hypothèse en termes d'internalisation des règles et des normes, elle propose des pistes de réflexion afin d'améliorer la formation du conducteur et sa prise en compte dans le continuum éducatif.

10 5. En décrivant l'organisation et les enjeux du continuum éducatif, Mariane Abramovici et Catherine Maman, mettent en avant l'intérêt de développer, de manière progressive, des compétences adaptées à chaque âge. Elles mettent l'accent sur la nécessité de développer une formation continue post-permis. Mais, de par la complexité de la tâche de conduite, la formation doit dépasser la simple acquisition de compétences techniques, il est vrai nécessaires, pour développer des compétences cognitives et comportementales. Ce travail passe obligatoirement par une observation de nos représentations sociales et par la compréhension de l'aspect social de la conduite automobile. Pour ces deux auteurs, le conducteur est pris en étau entre des choix de comportements solitaires et entre le respect de règles collectives et sociales, que cela soient dans nos choix de déplacements ou dans nos conduites à risque.

11 L'éducation routière pourrait être ainsi un moment de socialisation et d'intégration des règles et des normes sociales. Par contre, la diversité des compétences et des enjeux à l'œuvre, dans la formation à la conduite et à la sécurité routières, justifient le besoin de renforcer la formation des formateurs, mais également l'animation et la coordination du réseau d'acteurs contribuant à cette formation.

12 Comme nous l'avons vu précédemment, la matrice GDE devient la référence européenne pour toute réflexion éducative ayant comme visée, l'apprentissage d'une conduite plus sûre. Le rapport GADGET (1999), d'où est issue cette matrice, préconise notamment le développement de plusieurs compétences essentielles, afin d'atteindre les niveaux supérieurs de cette matrice. Ces compétences, centrées sur la personne, passent par la connaissance de soi, le changement identitaire et par de nouveaux comportements adoptés. Une des difficultés de cette approche consiste à déterminer quelles sont les approches et méthodes pédagogiques les plus à même à développer ces compétences du conducteur. Mais aucune amélioration ne peut être envisagée si les pratiques des enseignants de la conduite automobile restent les mêmes. 
13 6. Dans ce document, Dominique Violet, nous présente une autre approche causale et systémique des infractions et accidents routiers. Il y décrit la circulation routière comme une organisation complexe que l'État tente de réglementer et de contrôler. Le conducteur y dispose d'une forme d'autonomie, c'est-à-dire une capacité à y faire des choix, mais dans des limites réglementaires posées. Face à ce système en perpétuel mouvement et dans certaines zones d'incertitudes (densité de la circulation, diversité des usagers...), le conducteur y est soumis à des choix complexes. Sans nier le statut de la faute (non-respect des règles...) du conducteur et sa nécessaire régulation étatique et éducative, il nous présente ici le conducteur commettant des erreurs de choix de nature éthique dans certaines situations complexes. Dès lors, comme le préconise la matrice GDE, c'est en travaillant sur les niveaux supérieurs de la matrice (notamment les valeurs), que nous pourrions agir sur les comportements routiers. Précisément, l'organisation d'un système de valeur cohérent avec la sécurité et circulation routière, la mise en avant de certaines valeurs plus adéquates pour notre sécurité, sont des axes à privilégier en Éducation Routière.

14 Pour Dominique Violet, Il s'agirait donc ici davantage d'éduquer à la prudence que de former à la sécurité, de développer davantage la valeur « prudence » que d'apprendre à gérer des situations à risque, et enfin d'éduquer au jugement et à l'évaluation afin de permettre à l'usager de faire des choix plus pertinents quant à sa sécurité.

15 Cette approche intéressante et innovante nous oblige à revisiter notre conception de la formation du conducteur.

16 7. À partir des cursus développés au sein du département des sciences de l'éducation d'Aix-Marseille Université où la pédagogie de l'alternance est le fondement du dispositif de formation, nous explorons (Christine Poplimont, responsable des cursus en Éducation et Sécurité Routière et Maéva Duchène) l'apport d'une pensée réflexive sur les pratiques professionnelles.

17 Nous mettons en évidence, que si la dynamique de groupe est une modalité pédagogique organisationnelle nécessaire, ce sont les relations pédagogiques et d'accompagnement qui sont porteuses de changement des apprenants. Nous démontrons aussi que les modifications de comportement ne s'apprennent pas de manière conceptuelle, mais s'éprouvent, se ressentent et se vivent.

Ce travail prend donc toute son importance au moment où nous nous posons les questions de savoir comment produire et accompagner des changements durables auprès de nos apprentis conducteurs.

Line Courteaud et Christian Roux proposent un point de vue historique de la dimension sociale de la conduite. Les auteurs nous montrent que la formation du conducteur n'est pas en adéquation avec les conditions actuelles de circulation et ils abordent l'insécurité routière de façon systémique sous l'angle Homme-Véhicule-Environnement (HVE). La sécurité routière ne relève pas seulement de la performance mais aussi du comportement du conducteur, les conducteurs doivent passer d'une logique de conduire à se conduire. 


\section{BIBLIOGRAPHIE}

Vial, M. (1997). L'auto-évaluation, entre auto-contrôle et auto-questionnement. Les Cahiers de l'année 1997, Université de Provence, Département des Sciences de l'éducation, 12, 143-203.

Ardoino, J., \& Berger, G. (1986). L'évaluation comme interprétation. In l'Évaluation au pouvoir, revue POUR, $\mathrm{n}^{\circ} 107$.

GADGET (1999). Guarding Automobile Drivers trough Guidance Education and Technology : Orienter les conducteurs automobiles par le contrôle, l'éducation et les nouvelles technologies.

Le Boterf, G. (2000). Construire les compétences individuelles et collectives. Paris : Editions d'Organisation.

Gregersen, N.P. (1996). Young drivers' overestimation of their own skill: An experiment on the relation between training strategy and skill. Linköping, Sweden: Swedish National Road and Transport Research Institute.

Joule, R.-V., \& Beauvois, J-L. (1990). Petit traité de manipulation à l'usage des honnêtes gens. Grenoble : PUG.

Observatoire National Interministériel de la Sécurité Routière (ONISR) 2011 Les accidents corporels de la circulation.

Vurpillot, E., \& Pêcheux, M. G. (1992). Socialisation. In Collectif (Ed.), Grand dictionnaire de psychologie. Paris : Larousse.

Wilde, G. J.S. \& Ackersviller, M.J. (1982). The effect of posting observed driver behaviour upon subsequent driver response: The case of moving speed. Report prepared for Transport Canada, Traffic Safety, July.

Wilde G. J.S. (1988). Risk homeostasis theory and traffic accidents: propositions, deductions and discussion of dissension in recent reactions. Ergonomics.

Wilde G. J.S. (2001) The theory of risk homeostasis: implications for safety and health. Risk Analysis.

\section{AUTEUR}

\section{CHRISTINE POPLIMONT}

Maître de conférences, HDR, Aix-Marseille Université, EA 4671 ADEF, ENS de Lyon, IFE 